\title{
Analyse realer 110-kV-Lastverläufe für die zeitreihenbasierte probabilistische Lastflussrechnung in der Netzplanung unter Berücksichtigung der neuen Kundengruppe Rechenzentren
}

T. Pfüller(D, J. Hanson

\begin{abstract}
Verteilnetzbetreiber sind in den vergangenen Jahren immer neuen Herausforderungen ausgesetzt worden. Zu nennen sind die Energiewende, die Mobilitätswende und die Digitalisierung. Einige Netzbetreiber erleben die Digitalisierung in Form von vermehrten Netzanschlussanfragen von Rechenzentren im Verteilnetz, deren Größenordnung sich jeweils zwischen 5 MVA und 300 MVA bewegt. Um eine erfolgreiche und schnelle Integration dieser neuen Kunden zu ermöglichen, reicht der konventionelle Ansatz zur Planung, Starklast ohne Einspeisung und Schwachlast mit maximaler Einspeisung gemäß VDE-AR-N 4121, nicht mehr aus bzW. hätte dieser Ansatz einen enormen Netzausbau für die Verteilnetze zur Folge. Probabilistische Ansätze können bei der Bewältigung dieser Herausforderung helfen und eine bedarfsorientierte Netzausbauplanung vorantreiben. Das setzt eine Auseinandersetzung mit den realen Lastverläufen im 110-kV-Verteilnetz voraus. Dieser Artikel gibt einen Überblick über die Analyse und Gruppierung realer Lastverläufe im 110-kV-Verteilnetz der Syna GmbH aus dem Jahr 2018. Die Besonderheiten der Lastverläufe von Rechenzentren und deren Auswirkungen auf die Netzplanung werden aufgezeigt. Hierdurch werden aufschlussreiche Erkenntnisse über den tagestyp- und jahreszeitabhängigen Lastverlauf gewonnen. Auch lässt sich eine sehr große Abweichung von ca. 25 \% zwischen Starklast- und Höchstlastfall, bezogen auf die Netzlast, identifizieren. Insbesondere der Starklastfall ist bei einigen Verteilnetzbetreibern als "Worst-Case-Szenario" in Gebrauch. Dies ist einer der Gründe, die es rechtfertigen, sich mit den zeitreihenbasierten probabilistischen Lastflussrechnungen auseinanderzusetzen, mit dem Ziel, Strategien zum Anschluss großer Leistungen zu entwickeln.
\end{abstract}

Schlüsselwörter: Verteilnetzplanung; Gruppierung von Lastprofilen; zeitreihenbasierte probabilistische Lastflussrechnungen

\begin{abstract}
Analysis of real $110 \mathrm{kV}$ load curves for time series based probabilistic load flow calculations in network planning, taking the new client group data centers into account.

In recent years, distribution grid operators have been confronted with new challenges. These include the energy transition, mobility transition, and digitalization. Some grid operators face digitalization in the form of an increase in requests regarding grid connection from data centers in the distribution grid, the size of which ranges between 5 MVA and 300 MVA respectively. In order to enable a successful and fast integration of these new clients, the conventional approach to planning, heavy load without feed-in and low load with maximum feed-in according to VDE-AR-N 4121, is no longer sufficient or would result in an enormous grid expansion for the distribution grids. Probabilistic approaches can help to handle this challenge and advance demand-based grid expansion planning. This requires an analysis of the real load curves in the $110 \mathrm{kV}$ distribution grid. This paper provides an overview of the evaluation and grouping of real load curves in the $110 \mathrm{kV}$ distribution grid of Syna GmbH from 2018. It demonstrates the characteristics of the load curves of data centers and their impact on grid planning and provides useful knowledge about the load curve, which is dependent on the type of day and the time of year. It also identifies a very significant deviation of approx. 25\% between the peak load case and the maximum load case in relation to the network load. The peak load case, in particular, is used by some distribution grid operators as a "worst-case scenario". This is one of the reasons that has motivated us to look into time series based probabilistic load flow calculations, with the aim of developing strategies to connect large loads.
\end{abstract}

Keywords: distribution network planning; grouping of load profiles; time series based probabilistic load flow calculations

Eingegangen am 12. Juli 2021, angenommen am 13. September 2021, online publiziert am 28. September 202 (C) The Author(s) 2021

\section{Einleitung}

Die Verteilnetzbetreiber stehen vor der Herausforderung, das 110kV-Netz für eine geänderte Erzeugungs- und Laststruktur langfristig zu planen. Gemäß der aktuellen Planungsgrundsätze für 110-kVNetze VDE-AR-N 4121 berücksichtigt die Netzplanung hauptsächlich den Starklast-, Schwachlast- und den Jahreshöchstlastfall für die jeweiligen Netzgruppen [1]. Das 110-kV-Netz der Syna ist geprägt von Lasten in Form von Haushalten, Bürogebäuden, einem Flugha-

Pfüller, Tim, Fachgebiet Elektrische Energieversorgung unter Einsatz Erneuerbarer Energien (E5), TU Darmstadt, Landgraf-Georg-Straße 4, 64283 Darmstadt, Deutschland (E-Mail: tim.pfueller@gast.tu-darmstadt.de); Hanson, Jutta, Fachgebiet Elektrische Energieversorgung unter Einsatz Erneuerbarer Energien (E5), TU Darmstadt, Darmstadt, Deutschland 
fen und produzierender Industrie. Aufgrund des dicht besiedelten Gebietes in der Region um Frankfurt am Main findet keine nennenswerte Einspeisung aus erneuerbaren Energien in die 110-kVNetzebene im Vergleich zum Bezug statt.

Seit einigen Jahren gibt es verstärkt Anfragen von Rechenzentren (RZ) - mit einem jeweiligen Leistungsbedarf zwischen 5 und 300 MVA im Netzgebiet der Syna westlich von Frankfurt am Main. Würde die Syna alle Netzanschlussanfragen in der vom Kunden gewünschten Leistung bedienen, resultierte dies in einer Überlastung von 34 der 49 Stromkreise in einem Bereich von 100 - $190 \%$ im n-1 Fall. Das hieße ca. 172 km Kabel- und Freileitungsstrecken müssten verstärkt werden. Der konventionelle Ansatz stößt hier an seine Grenzen. Ein Netzausbau in dieser Größenordnung lässt sich nicht in absehbarer Zeit umsetzen.

Um die Netzplanung bedarfsgerecht voranzutreiben, werden die Messwerte eines gesamten Jahres analysiert und eine Gruppierung der Lastprofile für die verschiedenen 110-/20-kV-Umspannanlagen (UA) durchgeführt. Eine bessere Aussage über das Lastverhalten der Verbraucher in der 110-kV-Netzebene ermöglicht die Minimierung des Simulationsaufwands für zeitreihenbasierte probabilistische Lastflussrechnungen.

Der Einsatz von nicht-deterministisch definierten Verfahren ist nach VDE-AR-N 4121 für die 110-kV-Netzplanung zulässig [1]. Das Zugrundelegen realer Netzzustände für die Netzplanung kann ein entscheidender Schlüssel zur optimierten Auslastung vorhandener Netzstrukturen sein und den Netzbetreibern bei der Erfüllung der Aufgabe des wirtschaftlichen Netzbetriebs unterstützen.

\subsection{Zeitreihenbasierte probabilistische Lastflussrechnungen} Bereits 1974 sind erste Ansätze zu probabilistischen Lastflussrechnungen veröffentlicht worden [2, 3]. Trotz dieser sehr langen Zeit haben die nicht-deterministischen Verfahren nicht vollständig Einzug in die Planungs- und Betriebsgrundsätze von 110-kV-Netzen gehalten.

Die VDE-AR-N 4121 führt als Nachteil für die probabilistischen Verfahren unter anderem auf, dass für die Simulation eine hohe Rechenleistung benötigt wird und aufgrund der großen Menge an Eingangs-und Ausgangsdaten spezielle Algorithmen notwendig sind [1].

Für die zeitreihenbasierten probabilistischen Lastflussrechnungen werden nicht einzelne Lastzeitpunkte für Extremszenarien, sondern Zeitreihen simuliert. Diese Zeitreihen bilden den tatsächlichen Lastverlauf der Last über einen definierten Zeitraum nach und werden in der Regel aus realen Messwerten gewonnen. Mittels Monte-CarloSimulation können theoretisch alle Lastverläufe der einzelnen Lasten miteinander kombiniert werden. Hierdurch entstehen neue Lastsituationen in der Netzgruppe auf Grundlage der realen Lastverläufe [4]. Nachteil der Monte-Carlo-Methode ist, dass theoretisch unendlich viele Wiederholungen notwendig sind, da der Ansatz auf dem Gesetz der großen Zahlen beruht [5]. Da dies nicht möglich ist, muss festgelegt werden, wann die Simulation abgebrochen wird. Dies birgt die Gefahr kritische Lastfälle nicht zu berücksichtigen. Durch die Reduzierung der Eingangsdaten bzw. der Lastprofile müssen weniger Simulationen durchgeführt werden, um alle Kombinationen zu erhalten oder sich einem Erwartungswert anzunähern.

Die Anzahl der möglichen Kombinationen hängt von der Anzahl der nachgebildeten Lasten und der berücksichtigten Lastprofile ab. Sie berechnet sich aus der Anzahl der Lastprofile potenziert mit der Anzahl vorhandener Umspannanlagen, welche jeweils mit einem Lastprofil nachgebildet werden. Die Netzgruppe der Syna setzt sich aus 29 Umspannanlagen zusammen. Um die Kombinationsanzahl zu reduzieren, ist die Anzahl der Lastprofile zu reduzieren. Dies wird mit einem Algorithmus zur Gruppierung der Lastprofile umgesetzt.
Tab. 1. Ergebnis der Sortierung der Tages- und Jahreszeittypen

\begin{tabular}{lllll}
\hline & Frühling & Sommer & Herbst & Winter \\
\hline Werktage & 61 & 67 & 63 & 60 \\
Samstage & 13 & 14 & 12 & 13 \\
Sonn- u. Feiertage & 18 & 13 & 13 & 16 \\
\hline
\end{tabular}

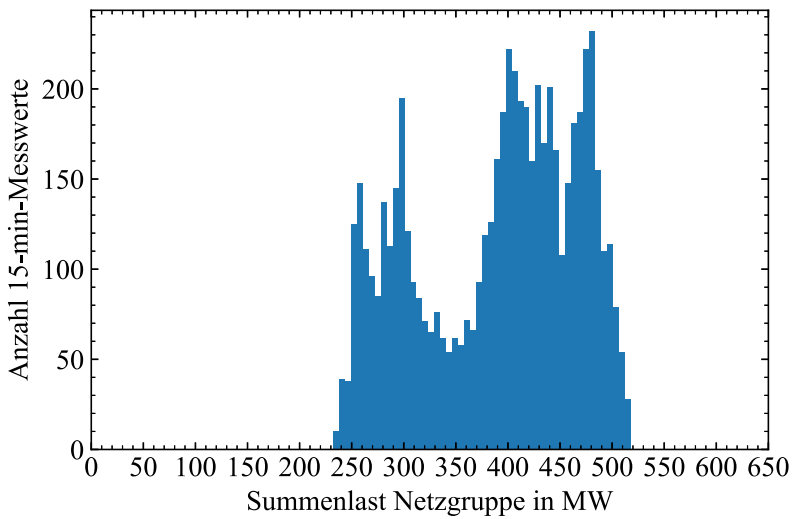

Abb. 1. Histogramm der Netzgruppenlast für Herbst-Werktage

Bevor eine Gruppierung durchgeführt wird, ist die Aufbereitung der Messwerte mit einer Abschätzung des möglichen Potenzials der zeitreihenbasierten probabilistischen Lastflussrechnung sinnvoll.

\section{Aufbereitung der Messwerte}

Die Messwerte liegen als 15-min-Mittelwertmesswerte der Wirkund Blindleistung für sämtliche 110-/20-kV-Transformatoren und $110-k V-K u n d e n$ vor. Diese werden in einem ersten Schritt nach Jahreszeit- und Tagestypen sortiert. Die drei Tagestypen sind Werktage, Samstage und Sonn- und Feiertage. Anschließend erfolgt eine Zusammenfassung der Lastprofile an den $U A^{2}$. Die resultierende Sortierung ist in Tab. 1 gezeigt. Die zwei Tage, welche die Zeitumstellung beinhalten, finden keine Berücksichtigung. Die Sortierung ermöglicht die spätere Berücksichtigung und Beurteilung von jahreszeit- und tagestypspezifischen Besonderheiten. Für die Potenzialabschätzung werden im nächsten Abschnitt die Lastzustände der Netzgruppe ausgewertet.

\section{Auswertung der Netzgruppe}

Die Netzgruppenlast setzt sich aus der Summe aller Lasten der 110kV-Netzgruppe zusammen. Mit dieser Auswertung soll die Frage beantwortet werden, wie häufig der Jahreshöchstlastfall auftritt und vor allem zu welcher Jahreszeit und welchem Tagestyp. Diese Frage lässt sich aus dem Histogramm der Netzgruppenlast beantworten, welches in Abb. 1 gezeigt ist. Der Abb. 1 ist zu entnehmen, dass die Jahreshöchstlast 520 MW beträgt. Der Starklastfall für das betrachtete Jahr beträgt $647 \mathrm{MW}$, welcher aus der zeitlich unabhängigen maximalen Bezugsleistung der einzelnen Lasten bestimmt wird und somit nicht im Histogramm ersichtlich ist, da dieser in der Realität nicht eintritt.

Des Weiteren ist aus Abb. 1 ersichtlich, dass diese Jahreshöchstlast ca. 30 mal im betrachteten Zeitraum auftritt. Das entspricht einer Zeit von 7,5 Stunden. Ob die Leistung für 7,5 Stunden über mehrere

${ }^{2}$ Die Lastprofile an einer UA setzen sich aus der Summe aller an dieser UA angeschlossenen Lasten zusammen. 
Tage verteilt oder am Stück vorliegt, lässt sich aus dem Histogramm nicht erkennen. In Abschn. 5 wird gezeigt, dass die Lastspitzen an den UA nur für einen kurzen Zeitraum auftreten. Dies lässt die Annahme zu, dass die Jahreshöchstlast über mehrere Tage verteilt und somit nicht für 7,5 Stunden am Stück bezogen wird.

Interessant ist die Differenz zwischen Höchst- und Starklast von ca. $127 \mathrm{MW}$. Dies entspricht einer Differenz von ca. $25 \%$ zwischen der Stark- und Jahreshöchstlast und deutet auf eine große Differenz zwischen den realen Netzsituationen und den für die Netzplanung angenommenen Szenarien hin.

Nachfolgend wird die Methode der Lastprofilgruppierung vorgestellt, welche auf dem Algorithmus von Wenyuan basiert [4]. Dieser verwendet die Fuzzy Logik nach Zedah [6].

\section{Methodik der Lastprofilgruppierung}

Der Ansatz der Gruppierung beruht auf der Winkeldifferenz der einzelnen Tageslastprofile an den jeweiligen UA. Hierbei handelt es sich um den geometrischen Winkel $\varphi$ zwischen zwei Tageslastprofilen in Vektorschreibweise. Vorangehend erfolgt die Sortierung gemäß Abschn. 2. Die Messwerte liegen als 15-min-Mittelwerte vor, sodass sich ein Taglastprofil aus 96 Messwerten zusammensetzt. Somit hat der Vektorraum eine Dimension von 96.

Das Grundprinzip berücksichtigt nachfolgende Relationsvorschrift: Der Cosinus des Winkels $\varphi$ zwischen zwei Vektoren $a$ und $b$ berechnet sich aus dem Skalarprodukt [7]:

$$
\cos (\varphi)=\frac{a \cdot b}{|a| \cdot|b|}
$$

Der $\cos (\varphi)$ wird zwischen allen Tageslastprofilen pro UA berechnet, sodass sich für jede UA eine quadratische Matrix $R$ ergibt, welche den Cosinus des Winkels zwischen den jeweiligen Profilen zueinander enthält. Der Cosinus bewegt sich in einem Wertebereich von 0 bis 1 [4]. Je ähnlicher der Verlauf von zwei Tageslastprofilen, desto näher ist der Wert des $\cos (\varphi)$ an Eins. Bei identischen Tageslastprofilen ergibt sich der Wert Eins, da die Winkeldifferenz in diesem Fall Null beträgt. Die Dimension $d \times d$ der Matrix $R$ entspricht der Anzahl der betrachteten Lastprofile. In der nachfolgenden Notation sind die Zeilen mit $n$ und die Spalten mit $m$ deklariert. Anschließend muss die für jede UA-Last berechnete Matrix auf Symmetrie, Reflexivität und Transitivität überprüft werden. Die drei Eigenschaften sind wie folgt definiert [4]:

1. Symmetrie

$$
R(\mathrm{~nm})=R(\mathrm{mn})
$$

2. Reflexivität

$$
R(\mathrm{~mm})=1
$$

3. Transitivität

$$
R \circ R \subset R
$$

Transitivität liegt vor, wenn durch gegenseitige Beziehung zwischen jeweils zwei Elementen auf die Beziehung zwischen allen Elementen und auf die Beziehung der Elemente mit sich selbst geschlossen werden kann. Die ermittelte Matrix $R$ enthält die Beziehungen in Form des $\cos (\varphi)$ der Tageslastprofile zueinander. Es müssen für die Transitivität somit auch Symmetrie und Reflexivität vorliegen. Diese liegt in der Regel nicht vor, kann aber mittels max-minKomposition hergestellt werden. [8]
Hierbei wird die ermittelte Matrix $R$ insgesamt $d-1$ mal mit sich selbst multipliziert. Allerdings ist anstatt der klassischen Berechnung des Matrizenprodukts die max-min-Komposition anzusetzen gemäß Gleichung 5. Das heißt, dass anstelle der komponentenweisen Multiplikation der Zeile $n$ mit der Spalte $m$ für jedes Komponentenpaar das Minimum ermittelt wird. Die anschließende Summation zur Ermittlung des Eintrages an der Stelle $R(\mathrm{~nm})$ der resultierenden Matrix $R$ wird durch die Ermittlung des Maximums ersetzt.

$$
(R \circ R)_{\mathrm{nm}}=\max _{\mathrm{k}} \min \left(R_{\mathrm{mk}}, R_{\mathrm{kn}}\right)
$$

Im nächsten Schritt des Gruppierungsalgorithmus erfolgt die Definition des Grenzwertes für die Gruppierung. Für das Winkelkriterium bilden alle Profile, die einen Zugehörigkeitswert größer $\cos \left(5^{\circ}\right)=$ 0,996 aufweisen, eine Gruppe. Es zeigt sich, dass ein Winkel von $5^{\circ}$ als Kriterium ausreicht.

Die ermittelten Werte in der Matrix $R$ beinhalten den Cosinus der jeweiligen Winkeldifferenz zwischen den Tageslastprofilen. Um eine Gruppierung vorzunehmen, muss ein Grenzwert definiert werden, welcher festlegt, zu welcher Gruppe ein Profil gehört. Je näher der gewählte Grenzwert an Eins ist, desto mehr Gruppen werden in der Regel entstehen, da die zulässige Winkeldifferenz zwischen den Profilen kleiner wird. Allerdings ist der Verlauf der Tageslastprofile innerhalb der jeweiligen Gruppe ähnlicher. Da das Ziel im Vordergrund steht die Anzahl der Eingangsdaten zu reduzieren, sollen im ersten Schritt der Gruppierung maximal zwei Gruppen entstehen. Mit der Wahl von $\cos \left(5^{\circ}\right)$ entsteht eine vorherrschende Gruppe von Tageslastprofilen, die einen sehr ähnlichen Verlauf haben und eine zweite Gruppe, die eine größere Abweichung von der vorherrschenden Gruppe vorweist. Die Feingruppierung erfolgt im nächsten Schritt mit dem Kriterium der maximalen Leistungsdifferenz von 5 MW der Tageslastprofile innerhalb der jeweiligen Gruppe. Die Leistungsdifferenz von $5 \mathrm{MW}$ wird ebenfalls anhand der Betrachtung der Ergebnisse der Tageslastprofilen für die 110-kV-Ebene gewählt. Hierfür wird in den beiden Gruppen jeweils das Tageslastprofil mit der größten Wirkleistung ermittelt. Anschließend wird geprüft, ob sich die einzelnen Tageslastprofile um mehr als 5 MW unterscheiden. Falls dies der Fall ist, bilden diese Tageslastprofile eine zweite Gruppe. Dieses Vorgehen wird für die zweite Gruppe wiederholt, nicht aber für eine mögliche dritte Gruppe. Der Verzicht auf eine weitere Feingruppierung der letzten Gruppe begründet sich damit, dass einmal vermieden werden soll weitere Gruppen zu erzeugen und zum anderen damit, dass diese Gruppe die Tageslastprofile mit den kleinsten Wirkleistungsverläufen beinhaltet. Somit können mit dem Gruppierungsalgorithmus maximal sechs Gruppen entstehen: Zwei Gruppen nach dem Kriterium der Winkeldifferenz und aus diesen jeweils maximal drei Gruppen nach dem Kriterium der Wirkleistungsdifferenz innerhalb der Gruppe.

Im letzten Schritt erfolgt die Aussortierung der Gruppen, welche eine Auftrittshäufigkeit von weniger als $5 \%$ und nicht die maximale Wirkleistung beinhalten. Dies führt zu einer Reduktion von 331.776 auf 864 Kombinationen. Die notwendige Simulationszeit für einen Computer mit mittlerer Ausstattung für die betrachtete Netzgruppe mit 864 Kombinationen beträgt ca. 20 Stunden.

\section{Auswertung der Lastverläufe}

Ausgewertet werden die einzelnen 110-kV-Lasten und die Netzgruppe separat für die jeweiligen Jahreszeiten und Tagestypen. Nachfolgend werden die Kategorien für "UA für Wohngebiete" und „UA für Rechenzentren" betrachtet. In diesem Artikel werden nur die Herbst-Werktage dargestellt, da in diesem Zeitraum die Jahreshöchstlast auftritt und so ein Vergleich zur konventionellen Netzplanung möglich ist. 


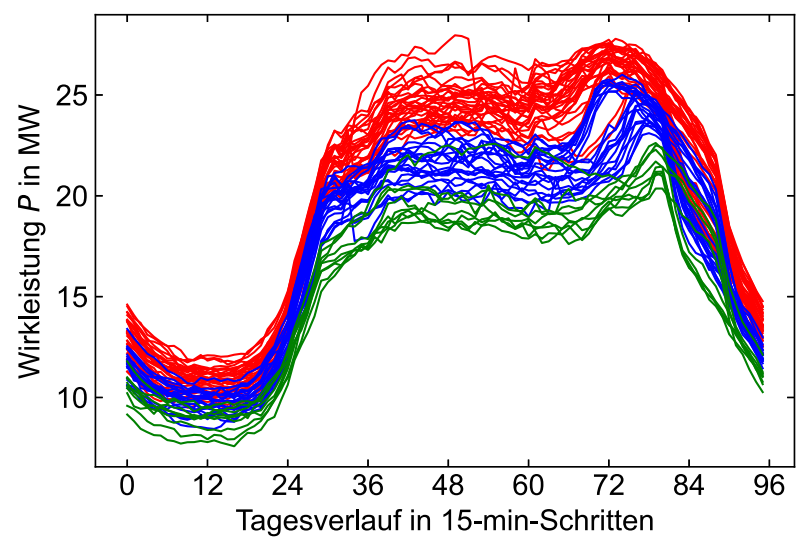

Abb. 2. Tageslastverläufe von Herbst-Werktagen der UA-Wohngebiet mit farblich gekennzeichneten Gruppen (Color figure online)

\subsection{Umspannanlage für ein Wohngebiet}

In Abb. 2 sind die Tageslastverläufe aller Herbst-Werktage für die UA-Wohngebiet dargestellt. Gemäß Tab. 1 sind 63 Tageslastverläufe gezeigt. Der erste Zeitpunkt 0 entspricht der Uhrzeit 0:15 Uhr. Da die Messwerte als 15-min-Mittelwerte vorliegen, setzt sich ein Tag aus 96 Messwerten zusammen. Diese UA versorgt hauptsächlich Haushalte und repräsentiert somit die Kategorie "Wohngebiete". Die Farbgebung kennzeichnet das Ergebnis der Gruppierung nach dem Leistungskriterium. Unter dem Leistungskriterium ist die im Abschn. 4 beschriebene Feingruppierung nach der maximalen Leistungsdifferenz von $5 \mathrm{MW}$ innerhalb der Gruppen zu verstehen. Da alle Profile eine kleinere Winkeldifferenz als $5^{\circ}$ zueinander haben, wird keine zweite Gruppe nach dem Winkelkriterium erstellt. Für Abb. 2 sind drei Gruppen erkennbar. Die Lastverläufe haben einen sehr ähnlichen Verlauf zueinander. Auch geht aus Abb. 2 hervor, dass die maximale Last im betrachteten Zeitraum nur sehr punktuell über einen kurzen Zeitraum auftritt. Der Zeitpunkt der Lastspitze liegt durchgehend bei ca. 19:00 Uhr (Zeitschritt 76).

\subsection{Umspannanlagen für Rechenzentren}

In Abb. 3 sind zwei RZ gezeigt: links RZ 1 mit einer installierten Leistung $P_{\text {inst. }}=90 \mathrm{MVA}$ und rechts RZ 2 mit $P_{\text {inst. }}=40 \mathrm{MVA}$. Für das linke RZ 1 sind zwei Gruppen (rot und blau) der Wirkleistungsverläufe erkennbar, welche sich aus der definierten Leistungsdifferenz ergeben. Der Verlauf zeigt einen homogenen Leistungsbezug. Der Blindleistungsbezug der RZ ist klein, dementsprechend wird auf die Darstellung der Blindleistungsverläufe verzichtet. Bei dem rechts gezeigten RZ 2 ist eine Gruppierung nach dem Winkelkriterium zu erkennen, hingegen liegt nur eine Gruppe für die Leistungsgruppierung vor. Die Wirkleistungsverläufe der zweiten Winkelgruppe sind gepunktet dargestellt und haben offensichtlich einen abweichenden Verlauf. Auffällig ist, dass der Wirkleistungsbezug für zwei Messpunkte bei Null liegt.

Bei der Betrachtung der RZ fällt eine Grundlast auf. Allerdings treten in beiden Fällen deutliche und meist kurzzeitige Sprünge in der Bezugsleistung bezogen auf die Grundlast auf. Dies gilt besonders für das RZ 2. Betrachtet man für beide RZ den Jahreslastverlauf, welcher in Abb. 4 dargestellt ist, lässt sich erkennen, dass Sprünge in der Bezugsleistung für RZ 2 (rechts) im gesamten Jahresverlauf auftreten. Auch ein Wachstumstrend ist zu erkennen. Im gesamten Jahr fällt die Bezugsleistung immer wieder auf Null. Mögliche Ursachen könnten ein Probebetrieb der Notstromgeneratoren oder Arbeiten an den Schaltanlagen sein.
Tab. 2. Entwicklung der mittleren und maximalen Bezugswirkleistung in MW der analysierten RZ seit Inbetriebnahme

\begin{tabular}{lllrrrrr}
\hline & 2011 & 2015 & 2016 & 2017 & 2018 & 2019 & 2020 \\
\hline$P_{1, \text { mittel }}$ & 16,7 & 16,1 & 19,7 & 25,4 & 33,2 & 36,3 & 39,8 \\
$P_{1, \text { max }}$ & 19,9 & 19,8 & 24,8 & 32,1 & 40,8 & 44,0 & 46,7 \\
$P_{2, \text { mittel }}-$ & - & 1,8 & 2,7 & 2,6 & 9,5 & 17,6 \\
$P_{2, \max }-$ & - & 3,3 & 5,4 & 5,6 & 13,7 & 21,9 \\
\hline
\end{tabular}

Das links dargestellte RZ 1 zeigt ebenfalls Sprünge in der Bezugsleistung, allerdings in einem deutlich geringeren Maße bezogen auf die Grundlast. Auch hier ist ein deutlicher Wachstumstrend zur Mitte des Jahres zu erkennen. Danach fällt die Bezugsleistung wieder ab. Der Rückfall könnte auf eine geänderte Nutzung hindeuten. Die betrachteten $R Z$ verhalten sich in ihrem Leistungsbezug unterschiedlich. Eine mögliche Ursache kann ein unterschiedliches Geschäftsmodell bzw. ein unterschiedlicher Kundenstamm sein. In Tab. 2 ist die jährliche Entwicklung der mittleren und maximalen Leistung seit Inbetriebnahme dargestellt. Man kann der Tabelle entnehmen, dass beide RZ über die Jahre kontinuierlich wachsen, aber weit entfernt von der installierten Leistung sind. Auch zeigt sich, dass die großen Sprünge in Abb. 4 in der Bezugsleistung von RZ 1 keine jahresspezifische Besonderheit darstellen, sondern in allen Jahren auftreten. Die maximal bezogene Leistung ist ca. $50 \%$ höher als die mittlere bezogene Leistung. Bei dem RZ 2 treten nur bis zu $20 \%$ Differenz auf.

Die große Differenz zwischen installierter und bezogener Leistung lässt sich unter anderem auf die zeitlich versetzten Bauabschnitte der Rechenzentren zurückführen. Die meisten neu geplanten Rechenzentren größerer Leistung unterteilen sich in mehrere Gebäude bzw. Blöcke, welche i.d.R einen Leistungsbedarf von 10 und 40 MVA pro Block aufweisen.

Betrachtet man das Jahr 2020, welches durch die CoronaPandemie geprägt wurde, fällt ebenfalls die unterschiedliche Entwicklung der beiden RZ auf. Für das RZ 1 ist ein kontinuierliches Wachstum in der Bezugsleistung und den Spitzen gegenüber dem Vorjahr zu erkennen. RZ 2 hingegen zeigt einen deutlichen Zuwachs in der Leistung. Diese hat sich gegenüber 2019 fast verdoppelt, allerdings lag ein starker Wachstumstrend bereits in 2019 vor.

\section{Ergebnisse und Diskussion}

Anhand des vorgestellten Gruppierungsalgorithmus können die Eingangsdaten signifikant reduziert werden, sodass im Rahmen der zeitreihenbasierten probabilistischen Lastflussrechnungen alle Kombinationen simuliert werden können. Die Anzahl der möglichen Kombinationen für die betrachtete Netzgruppe bei HerbstWerktagen konnte durch die umgesetzte Gruppierung von 331.776 auf 864 mögliche Kombinationen von Tageslastverläufen reduziert werden. Des Weiteren kann gezeigt werden, dass eine Differenz von $25 \%$ (127 MW) der Netzgruppenlast zwischen Jahreshöchstlastfall und Starklastfall vorliegt. Das mögliche Potenzial, welches durch die zeitreihenbasierten probabilistischen Lastflussrechnungen gehoben werden kann, ist ein gutes Argument, um diesen Ansatz zu implementieren. Der Jahreshöchstlastfall tritt nur über 7,5 Stunden im betrachteten Jahr auf. Der Starklastfall liegt zu keinem Zeitpunkt vor.

Es wird gezeigt, dass die analysierten RZ nur eine maximale Auslastung von ca. $50 \%$ erreichen. RZ 1 hat diesen Auslastungsgrad erst 9 Jahre nach der Inbetriebnahme erreicht, RZ 2 nach 4 Jahren. Durch die große Differenz zwischen Starklastbezug der RZ und 

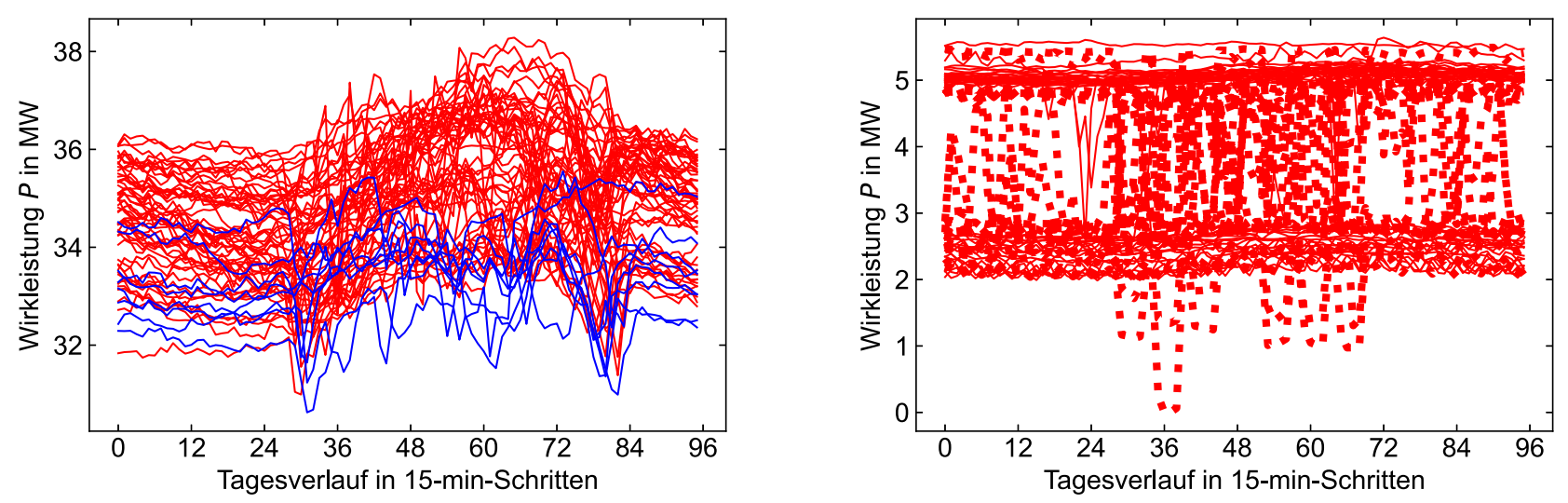

Abb. 3. Tageslastverläufe von Herbst-Werktagen von RZ 1 (links) und RZ 2 (rechts) (Color figure online)
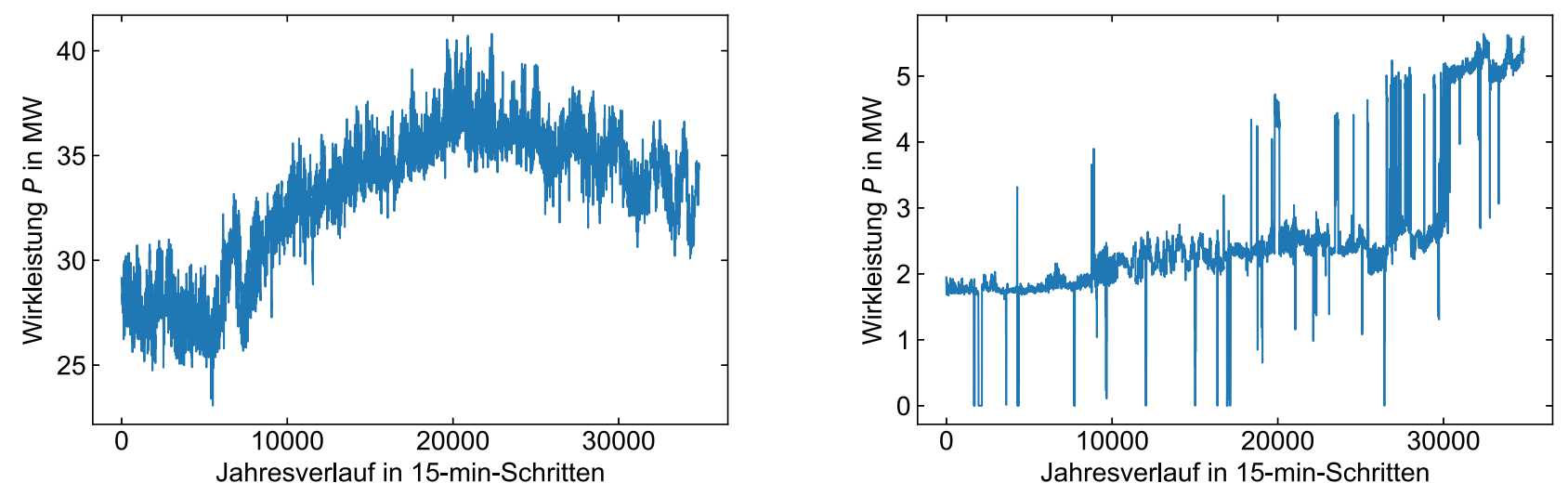

Abb. 4. Jahreslastverläufe von RZ 1 (links) und RZ 2 (rechts)

vertraglich kontrahierter Leistung $\left(=P_{\text {inst. }}\right)$ kann es zur Überdimensionierung des Verteilnetzes kommen. Die Berücksichtigung vieler RZ mit ihrer vertraglichen Leistung hat zur Folge, dass die Differenz zwischen Jahreshöchstlast und Starklast weiter zunimmt, da Kunden mit der vertraglichen Leistung in den Netzberechnungen berücksichtigt werden müssen. Hierdurch würde sich der Starklastfall im Netz der Syna von 647 MW auf 730 MW erhöhen.

\section{Schlussfolgerungen}

Rechenzentren unterscheiden sich im Lastverlauf und in den Wachstumsraten. Ein Standardlastprofil, wie es für Gewerbekunden existiert, ist nicht ohne Weiteres anwendbar. Auf Grundlage der gewonnenen Erkenntnisse über das Lastverhalten von RZ können mit den neu geplanten Rechenzentren sogenannte Stufenfunktionen vereinbart werden. Diese beinhalten eine schrittweise Erhöhung der kontrahierten Leistung, welche das jeweilige RZ beziehen darf und ist an den aktuellen Leistungsbezug und den eventuell notwendigen Netzausbau geknüpft. Somit wird keine Kapazität im Netz durch Kunden blockiert, welche diese erst perspektivisch benötigen. Um eine wirtschaftliche Netzplanung voranzutreiben, sollten probabilistische Ansätze stärker bei den Regelwerken und Verteilnetzbetreibern implementiert werden, mit dem Ziel, die Kapazitäten der bestehenden Netzinfrastruktur optimal auszunutzen und den Netzausbau zielgerichtet zu gestalten, ohne die betrieblichen Reserven zu vernachlässigen.

\section{Danksagung}

Diese Arbeit wurde im Rahmen des Forschungsprojekts zur probabilistischen Netzplanung mit dem Verteilnetbetreiber Syna GmbH aus Frankfurt am Main durchgeführt.

Funding Note Open Access funding enabled and organized by Projekt DEAL.

Conflict of interest The authors declare that they have no conflict of interest.

Hinweis des Verlags Der Verlag bleibt in Hinblick auf geografische Zuordnungen und Gebietsbezeichnungen in veröffentlichten Karten und Institutsadressen neutral.

Open Access Dieser Artikel wird unter der Creative Commons Namensnennung 4.0 International Lizenz veröffentlicht, welche die Nutzung, Vervielfältigung, Bearbeitung, Verbreitung und Wiedergabe in jeglichem Medium und Format erlaubt, sofern Sie den/die ursprünglichen Autor(en) und die Quelle ordnungsgemäß nennen, einen Link zur Creative Commons Lizenz beifügen und angeben, ob Änderungen vorgenommen wurden. Die in diesem Artikel enthaltenen Bilder und sonstiges Drittmaterial unterliegen ebenfalls der genannten Creative Commons Lizenz, sofern sich aus der Abbildungslegende nichts anderes ergibt. Sofern das betreffende Material nicht unter der genannten Creative Commons Lizenz steht und die betreffende Handlung nicht nach gesetzlichen Vorschriften erlaubt ist, ist für die oben aufgeführten Weiterverwendungen des Materials die Einwilligung des jeweiligen Rechteinhabers einzuholen. Weitere Details zur Lizenz entnehmen Sie bitte der Lizenzinformation auf http://creativecommons.org/licenses/by/4.0/deed.de. 


\section{Literatur}

1. VDE-Anwendungsregel VDE-AR-N 4121:2018-04, Planungsgrundsätze für 110-kVNetze.

2. Borkowska, B. (1974): Probabilistic load flow. IEEE Trans. Power Appar. Syst., 3, 752-759.

3. Allan, R. N., Borkowska, B., Grigg, C. H. (1974): Probabilistic analysis of power flows. Proc. Inst. Electr. Eng., 121, 1551-1556.

\section{Autoren}

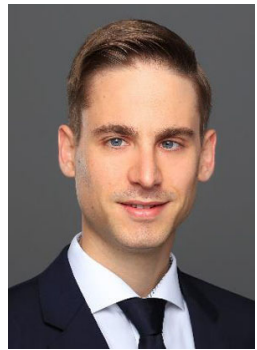

Tim Pfüller

absolvierte sein Studium der "Nachhaltigen Elektrischen Energieversorgung" an der Universität Stuttgart und ist seit 2016 bei der Syna $\mathrm{GmbH}$ in Frankfurt am Main beschäftigt. Nach einem Trainee-Programms im Asset Management Hochspannung arbeitete er als "Senior Projektmanager Digitalen Systemtechnik". Seit 2019 beschäftigt er sich mit der 110-kV-Zielnetzplanung bei der Syna $\mathrm{GmbH}$ in Frankfurt. Im gleichen Jahr nahm er seine externe Promotion am Fachgebiet E5 mit dem Schwerpunkt der probabilistischen Lastflussrechnungen auf.
4. Wenyuan, L. (2011): Probabilistic transmission system planning (S. 40-42). Hoboken: Wiley. S. 55-57 u. 327-328

5. Nahrstedt, H. (2015): Die Monte-Carlo-Methode: Beispiele unter Excel VBA (S. 2). Wiesbaden: Springer

6. Zadeh, L. A. (1965): Fuzzy sets. Inf. Control, 8, 338-353.

7. Bronstein, I. N., et al. (2012): Taschenbuch der Mathematik (Bd. 1, S. 361). Berlin: Springer.

8. Zadeh, L. A. (1971): Similarity relations and fuzzy orderings. Inf. Sci., 3(2), 177-200.

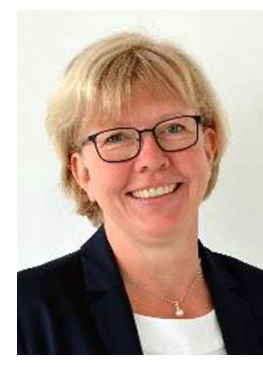

\section{Jutta Hanson}

hat an der Technischen Universität Darmstadt studiert und wurde dort im Jahr 2000 promoviert. Sie arbeitete bis 2011 bei der ABB AG in Mannheim in der Abteilung Elektrische Systemberatung. Hier leitete sie die Gruppe Übertragungs- und Industrienetze. Seit Oktober 2011 ist sie als Professorin für das Fachgebiet Elektrische Energieversorgung unter Einsatz Erneuerbarer Energien an der TU Darmstadt tätig. Ihre Forschungsschwerpunkte liegen im Bereich der Stabilität elektrischer Netze im Kontext eines zunehmenden Anteils umrichterbasierter Erzeugung und der Regelung von hybriden AC/DC-Netzen. Zudem beschäftigt Sie sich mit der Verteilnetzplanung unter Berücksichtigung energieoptimierter Quartiere. 